\title{
Value of testing glycated hemoglobin in non-diabetic patients presenting with acute coronary syndrome
}

\author{
P G C J Nanayakkara ${ }^{1}$, T P Weerarathna ${ }^{1}$, H M M Herath ${ }^{1}$, A G T A Kariyawasam ${ }^{1}$, V R Ganegoda ${ }^{1}$ \\ Sri Lanka Journal of Diabetes, Endocrinology and Metabolism 2015; 5: 4-7
}

\begin{abstract}
Objectives: To estimate the proportion of patients with no previous diagnosis of diabetes admitted with acute coronary syndrome having normal, impaired fasting glucose and diabetes on fasting blood glucose testing and normal, pre-diabetes and diabetes on glycated hemoglobin. To compare the clinical profiles of patients with acute coronary syndrome with no diabetes according to both tests, with those having no diabetes on fasting blood glucose but having diabetes according to glycated hemoglobin.

Methods: In a cross sectional study, fasting blood sugar and glycated hemoglobin were estimated in 125 patients without a previous history of diabetes admitted with acute coronary syndrome. Glycemic category was ascertained according to fasting blood glucose and glycated hemoglobin levels recommended by the American Diabetes Association.

Results: According to glycated hemoglobin testing, $47 \%$ and $53 \%$ of patients with acute coronary syndrome had diabetes and pre diabetes respectively. The corresponding percentages in each category according to fasting blood glucose testing were six (4\%) and twenty (16\%). There were no significant differences in the mean age, body mass index or waist circumference of patients in the categories with no diabetes on both testing methods and diabetes only on glycated hemoglobin testing.

Conclusions: According to glycated hemoglobin testing, every patient with acute coronary syndrome has either diabetes or pre diabetes. Compared to fasting blood glucose testing alone, additional testing for glycated hemoglobin in patients with acute coronary syndrome increase the number of patients with newly diagnosed diabetes by eight fold and pre diabetes by two and a half fold.
\end{abstract}

Key-words: Glycated hemoglobin, acute coronary syndrome, Pre-diabetes, Diabetes.

\section{Introduction}

Coronary artery disease (CAD) affects individuals with type 2 diabetes more frequently than those without diabetes (1). Acute coronary syndrome (ACS) represents a major clinical presentation of CAD. Studies reveal a positive correlation between the admission plasma glucose level and morbidity and mortality from CAD $(2,3)$. Individuals with diabetes benefit from more intensive and aggressive management protocols such as early referral for coronary angiography and preferential use of coronary artery bypass surgery over coronary angioplasty compared to those without diabetes (4).

Fasting blood glucose (FBS) is used to verify the glycaemic status of both diabetic and non-diabetic patients admitted to hospital with ACS. Occurrence of stress hyperglycemia and test result values in the range of impaired fasting glucose (100-125 mg/dL) limits its specificity in detecting the correct glycaemic status among individuals with no history of diabetes presenting with
ACS. Thus, in patients with ACS and previously undiagnosed diabetes, testing only for FBS could yield false positive or false negative results, depriving them of being managed according to their correct glycaemic category with the most optimal management protocol.

Recent guidelines recommend testing of glycated hemoglobin (HbA1c) level to diagnose pre-diabetes and diabetes (5). Individuals with $\mathrm{HbA} 1 \mathrm{c}>6.5 \%$ are classified as having diabetes and those with HbA1c between 5.6$6.4 \%$ are categorized having pre-diabetes. Testing for HbA1c does not require fasting, and it reflects glycaemic load over a longer period (three to four months). It is a more specific test than fasting plasma glucose in the diagnosis of the underlying glycaemic status.

Testing of HbA1c level in previously undiagnosed diabetic patients with ACS could help in detecting the correct glycaemic category than testing FBS alone. More specific information on the correct glycaemic category in

${ }^{1}$ Department of medicine, faculty of medicine, university of Ruhuna, Sri Lanka. 
the patients with ACS could supplement therapeutic decision making and potentially improve clinical outcomes. We planned to study the clinical utility of testing for HBA1c in detecting pre-diabetes and diabetes among patients without the previous diagnosis of diabetes mellitus admitted to hospital with acute coronary syndromes.

The main objectives of this study were

1. To assess the proportion of patients with no previous diagnosis of diabetes mellitus admitted with acute coronary syndrome having normal, impaired fasting blood glucose and diabetes on FBS and normal, prediabetes and diabetes on $\mathrm{HbAlc}$.

2. To compare the clinical profiles of patients with ACS with no diabetes on both FBS and HbAlc with those having no diabetes on FBS but HbA1c above the diabetic cut-off value.

\section{Methodology}

This is a cross sectional study conducted in a medical ward of a tertiary care hospital in Sri Lanka. We used convenient sampling method to include all male and female patients with no previous history of diabetes, admitted over a period of three months with any of the three types of acute coronary syndromes; ST elevation myocardial infarction, non-ST elevation myocardial infarction and unstable angina. Patients with previously diagnosed diabetes and those with a history of steroid use during the past three months were excluded.

We obtained data on age, gender, body mass index (BMI), waist circumference, drug treatment for previously diagnosed dyslipidemia, hypertension or coronary artery disease from the bed head tickets. Testing for $\mathrm{HbAlc}$ was done from the same sample of blood obtained for fasting blood glucose on the second day after admission. High performance liquid chromatography (HPLC) method was used to estimate $\mathrm{HbA} 1 \mathrm{c}$ level. Diagnostic cut-off values laid down by the American Diabetes Association for using $\mathrm{HbA} 1 \mathrm{c}$ to detect pre-diabetes and diabetes were used for categorization of glycaemic status.

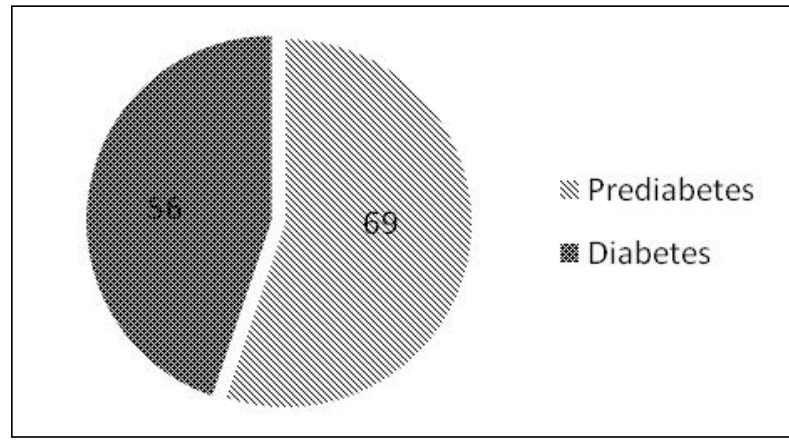

Figure 1. Number of patients with pre-diabetes and diabetes according to HbA1c

\section{Statistical analysis}

All numerical data were represented as mean (SD) and categorical data as proportions. Student's t test was used to compare the differences between numerical data and chi-squared test was used for the categorical data and level of 0.05 was considered as statistically significant.

\section{Results}

There were 64 males $(51 \%)$ in the study sample. Majority $(83 / 125,66 \%)$ had unstable angina and $20 \%$ and $14 \%$ were diagnosed as non-ST elevation and ST elevation myocardial infarction, respectively. Descriptive data on the study sample are shown in table1.

Table1. Descriptive statistics of study participants

\begin{tabular}{ll}
\hline & Mean $(\mathrm{SD})$ \\
\hline Age (years) & $63.3(13.4)$ \\
Body mass index $\left(\mathrm{Kg} / \mathrm{m}^{2}\right)$ & $21.9(4.3)$ \\
Waist circumference $(\mathrm{cm})$ & $81.4(12.2)$ \\
FBS $(\mathrm{mg} / \mathrm{dL})$ & $91.1(26.9)$ \\
$\mathrm{HbA1c} \%$ & $6.5(0.62)$ \\
\hline
\end{tabular}

Forty two (33\%) patients gave a previous history of $\mathrm{CAD}$, and the percentage of patients under antihypertensive and lipid lowering therapy were $47 \%$ and $34 \%$ respectively. Family history of diabetes was elicited in $23 \%$, and $35 \%$ were current smokers.

Of the 125 patients studied, $99(80 \%)$ had normal blood glucose $(<125 \mathrm{mg} / \mathrm{dL}), 20$ (16\%) had impaired fasting glucose (IFG) and $6(4 \%)$ were newly diagnosed to have diabetes, based on FBS. HbAlc levels of all 6 newly diagnosed patients with diabetes according to FBS were above $7 \%$ and ranged from $7-8.9 \%$ (Figure 1 and 2).

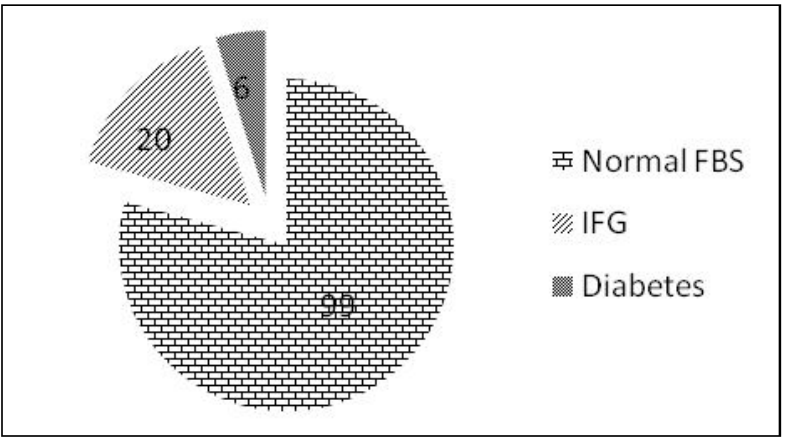

Figure 2. Number of patients in different glycemic categories according to FBS 
Based on HbA1c, 59 (47\%) had diabetes and 66 (53\%) were in the category of pre-diabetes. None of the study subjects had HbA1c level below 5.5\% to be categorized as having normal blood glucose. Out of the 66 in the prediabetes category, only 7 subjects had FBS in the range of IFG, leaving 59 (89\%) patients with pre-diabetes on HbA1c having normal FBS (<100 mg / dL).

Number of patients in the three different glycemic categories (normal, impaired fasting glucose/ prediabetic and diabetes) with FBS and HbA1c is shown in the table 2.

Table 2. Number of patients in different glycemic categories

\begin{tabular}{lll}
\hline & FBS & HbA1c \\
\hline Normal & 99 & None \\
IFG / Pre-diabetes & 20 & 69 \\
Diabetes & 6 & 56 \\
\hline
\end{tabular}

Out of the 119 patients with FBS in the non-diabetic range ( $<125 \mathrm{mg} / \mathrm{dL}$ ), 50 were categorized having diabetes according to $\mathrm{HbA} 1 \mathrm{c}(>6.5 \%)$ and 69 were in the pre diabetic range (5.5- 6.5\%).

Comparison of the clinical profiles of patients with no-diabetes on both tests and those with no diabetes on FBS but diabetes on HbA1c is shown in the table 3 .

\section{Table 3. Comparison of patients with no diabetes by both tests vs diabetes on HbA1c with no diabetes by FBS}

\begin{tabular}{llll}
\hline & $\begin{array}{l}\text { No diabetes on } \\
\text { both FBS \& } \\
\text { HbA1c }\end{array}$ & $\begin{array}{l}\text { No diabetes on } \\
\text { FBS but diabetes } \\
\text { on HbA1c }\end{array}$ & P value \\
\hline $\begin{array}{l}\text { Number of } \\
\text { patients }\end{array}$ & 69 & 50 & \\
$\begin{array}{l}\text { Age in years } \\
\text { (mean } \pm \text { SD })\end{array}$ & $62.41(14.32)$ & $64.02(12.51)$ & $0.52^{*}$ \\
$\begin{array}{l}\text { BMI Kg/m }{ }^{2} \\
\text { (mean } \pm \text { SD) }\end{array}$ & $22.42(4.46)$ & $20.95(4.07)$ & $0.06^{*}$ \\
$\begin{array}{l}\text { Waist } \\
\text { circumference } \\
\text { CM (mean } \pm \text { SD) }\end{array}$ & $80.77(13.00)$ & $82.15(11.61)$ & $0.56^{*}$ \\
$\begin{array}{l}\% \text { with } \\
\text { complications } \\
\text { during hospital } \\
\text { stay }\end{array}$ & $18 \%$ & & \\
\hline
\end{tabular}

*student t test, ${ }^{\#}$ chi $^{2}$ test.

\section{Discussion}

We found that every patient without previous history of diabetes presenting with ACS has either diabetes (47\%) or pre diabetes (53\%) according to HbA1c. Altogether 59 patients were found to have diabetes on HbA1c as opposed to 6 patients on FBS. All the 6 patients who had diabetic FBS had HbA1c above the diabetic range. HbA1c detected an additional 50 patients as having diabetes among the 119 with FBS in the non-diabetic range. There was an eight-fold increase (6 vs 50) in the number of diabetics when HbA1c was used as the diagnostic criteria as opposed to FBS. Proportion with pre-diabetes was also two and a half times more when HbA1c was used as the method of diagnosis than when FBS was used.

We used HbA1c cut-off points recommended by the American Diabetes Association for the diagnosis of diabetes and pre diabetes. Studies have reported variations in the HbA1c levels in different ethnicities and some have recommended higher HbA1c cut-off values for the categorization of pre-diabetes and diabetes (6). Our findings could have been different had we known the ethnic specific HbA1c for our population.

As a diagnostic tool, HbA1c is less sensitive and more specific than FBS in detecting diabetes and pre diabetes, hence its use may be associated with an over diagnosis of individuals with both conditions (6). But considering the rising incidence of diabetes in the Sri Lankan community and the relatively high mean age (62 years) of patients included in our study, the $43 \%$ prevalence of diabetes and $57 \%$ of pre diabetes is not surprising and it only strengthens the notion that that type 2 diabetes is cardiovascular disease (7).

Prevalence of glucose abnormalities among patients with no previous history of diabetes presenting with ACS in previous studies vary between $21 \%$ - $84 \%(8,9)$. A study conducted in urban India revealed that $84 \%$ of patients with ACS with no previous history of diabetes have abnormalities in glucose metabolism based on the findings of OGTT (10). Using HbA1c cut-off level of 6.5\%, the prevalence of diabetes in that study was $25 \%$. The mean age of their study sample is lower than in our study (55 vs 62 years) and it is well established that the prevalence of diabetes increases with age. Another group that aimed to define the acceptable HbA1c cut-off value to recognize individuals with pre-diabetes and diabetes in the setting of ACS have reported that HbA1c level of 6.7\% detects diabetes with $85.45 \%$ sensitivity and $91.89 \%$ specificity (11).

Based on the findings in several studies showing high rates of prevalence of glucose abnormalities among patients with ACS, Gholap and coworkers have drawn-up a simple algorithm to identify the correct glycemic category and implement appropriate management protocols for 
these patients (12). According to their algorithm, HbA1c should be done in everyone with ACS. Patients with ACS and no previous history but symptoms of diabetes, and with HbA1c of over $6.5 \%$ should be managed as having diabetes. For the same category without symptoms repeating the test in 8 weeks is recommended and they too should be managed as diabetes if the repeat HbA1c exceeds $6.5 \%$. For those with repeat value between $6.4 \%$ - 6.2\%, they recommend OGTT. And those with post discharge $\mathrm{HbA} 1 \mathrm{c}<6.2 \%$, annual screening for diabetes is recommended. Cost effectiveness and the short and long term clinical outcome of this approach has not been elucidated. But this approach ensure giving due recognition for high prevalence of glucose abnormalities among patients presenting with ACS and obviate the need for OGTT for each and every patient found to have borderline HbA1c in the setting of an ACS.

We found that the additional 50 patients with diabetes according to HbA1c but with non-diabetic FBS were older and had higher waist circumferences and lower BMI when compared to 69 patients who had no diabetes on both FBS and HbA1c. It implies that older patients and those with central but not global obesity without a previous diagnosis of diabetes are more likely to be detected by testing for their HbA1c than FBS. But lack of statistical significance between the studied variables precludes us from arriving at such conclusions.

Finding of eight-fold (6 vs 50) increase of number of patients with diabetes and two and a half fold increase in the category with pre-diabetes by testing for HbA1c compared to FBS among patients in this study challenges the diagnostic utility of FBS in correctly recognizing the glycaemic status of individuals presenting with acute vascular catastrophes such as acute coronary syndrome. It exposes the need for a test with more sensitivity and specificity. Findings of our study may not be compelling at present for clinicians to test HbA1c in each and every patient with ACS with no previous history of diabetes, but they serve as an eye opener of higher prevalence of glucose abnormalities among patients presenting with ACS in the Sri Lankan setting.

There are few limitations in our study. We used convenient sampling method to recruit patients and there is a substantial majority with unstable angina than the other two categories of ACS in the study sample. Had we included only the patients with ST elevation infarctions the results may have been different due to more robustness in the diagnosis of ST elevation MI compared to unstable angina. The other limitation in this study is that we did not verify the glycemic status of patients included with a subsequent OGTT. Nevertheless the long held notion that "type 2 diabetes is a cardiovascular disease" is aptly endorsed by the findings of our study. As the study was conducted in a medical ward rather than in a coronary or intensive care unit, the recruited patients were stable. Failure to find any patient with stress hyperglycemia
(FBS > $126 \mathrm{mg} /$ dLwith HbA1c < 6.5\% ) may be due to the inclusion of clinically stable patients with acute coronary syndrome in this study and the relatively small sample size.

In conclusion, we report nearly a 50\% prevalence of diabetes and pre-diabetes according to HbA1c among patients with no previous history of diabetes presenting with ACS. We emphasize the need for detecting correct glycemic category of these patients using a better diagnostic tool than FBS. Whether or not testing of HbA1c in this category of patients could fill this gap need to be investigated by further studies including a gold standard test such as OGTT for the diagnosis of different glycemic categories.

\section{References}

1. Zhang PY. Cardiovascular disease in diabetes. European review for medical and pharmacological sciences 2014; 18 (15): 2205-14.

2. Giraldez RR, Clare RM, Lopes RD, et al. Prevalence and clinical outcomes of undiagnosed diabetes mellitus and prediabetes among patients with high-risk non-ST-segment elevation acute coronary syndrome. Am Heart J 2013; 165(6): 918-25.e912.

3. Naito R, Miyauchi K, Ogita M, et al. Impact of admission glycemia and glycosylated hemoglobin A1c on long-term clinical outcomes of non-diabetic patients with acute coronary syndrome. J Cardiol 2014; 63(2): 106-11.

4. Executive summary: Standards of medical care in diabetes 2014. Diabetes Care 2014; 37 Suppl 1: S5-13.

5. Bennett CM, Guo M, Dharmage SC. HbA(1c) as a screening tool for detection of Type 2 diabetes: a systematic review. Diabet Med 2007; 24(4): 333-43.

6. John WG. Use of HbA1c in the diagnosis of diabetes mellitus in the UK. The implementation of World Health Organization guidance 2011. Diabet Med 2012; 29(11): 1350-7.

7. Jayawardena R, Ranasinghe P, Byrne NM, et al. Prevalence and trends of the diabetes epidemic in South Asia: a systematic review and meta-analysis. BMC Public Health 2012; 12: 380.

8. Abdullatef WK, Al-Aqeedi RF, Dabdoob W, et al. Prevalence of unrecognized diabetes mellitus in patients admitted with acute coronary syndrome. Angiology 2013; 64(1): 26-30.

9. Chan CY, Li R, Chan JY, et al. The value of admission HbA(1c) level in diabetic patients with acute coronary syndrome. Clinical Cardiology 2011; 34(8): 507-12.

10. Ramachandran A, Chamukuttan S, Immaneni S, et al. High incidence of glucose intolerance in Asian-Indian subjects with acute coronary syndrome. Diabetes Care 2005; 28(10): 2492-6.

11. Caldeira D, de Castro Linhares A, Bettega M, et al. A1C as a diabetes diagnosis method: experience in a coronary ICU. Journal of Cardiovascular Medicine (Hagerstown, Md) 2013; 14(11): 827-32.

12. Gholap N, Davies MJ, Mostafa SA, et al. A simple strategy for screening for glucose intolerance, using glycated haemoglobin, in individuals admitted with acute coronary syndrome. Diabet Med 2012; 29(7): 838-43. 